\title{
A very cool birthday
}

\author{
Superconductivity may have reached its centenary, but if anything it's a more active field of research \\ today than ever. From materials dull or shiny, to the race for the Higgs boson, superconductivity remains \\ relevant and exciting.
}

These days, cryogenic liquids are as ubiquitous as the celebrity chefs who use them to make ice cream and other frozen delights. Thus has the dream of Heike Kamerlingh Onnes (pictured) come to pass, for liquid helium to be "transported just as the other liquid gases now are, and in which this gas also, one might say, will be as freely available as water"'. Well, nearly. But around the time that Kamerlingh Onnes was attempting to liquefy helium-4, even such innovators as James Dewar - who liquefied hydrogen and invented the portable vacuum flask that bears his name - had failed at the task and thought it to be impossible.

Undaunted by the naysayers and driven by his passion to test his colleague Johannes van der Waals's thermodynamic theories, Kamerlingh Onnes succeeded in liquefying helium in 1908 - a

feat that brought scientists five times closer to absolute zero. It also enabled Kamerlingh Onnes to measure the lowtemperature resistance of metals. Based on higher-temperature data, he assumed that the resistance of pure metals (gold, for example) would smoothly approach zero at $T=0$. In the case of very pure mercury, he was correct in his assumption, except for the shocking abruptness of the transition to zero resistivity at $4.2 \mathrm{~K}$. He had discovered a new state of matter - a macroscopic quantum state.

At 100 years old this month, superconductivity is everywhere. Lead, tin, aluminium, uranium, even dirty ceramic pellets - all superconduct below a certain temperature. Applications based on wires with zero dissipation are still, however, limited to low temperatures. But without superconductivity, the Large Hadron Collider wouldn't be possible: it relies on superconducting cable, wound into thousands of dipole and quadrupole magnets and bathed in tonnes of liquid helium, to steer and focus its proton beams. More every-day applications include magnetically levitated trains, electrical cables, sensitive magnetic field sensors (SQUIDs) and more. (Our sister title Nature Materials offers a wider survey of the applied side of superconductivity in its April issue ${ }^{2}$.)

To describe conventional superconductors, which are mostly elements, we have the microscopic model published by John Bardeen, Leon Cooper and Robert Schrieffer in 1957. Their major insight was that the electrons are paired up by means of a phonon 'glue', and the phenomenon of superconductivity was considered solved. But in 1986 the field experienced another quantum leap when the copper-oxide superconductors (cuprates) burst onto the scene - long have they reigned as recordholders, having transition temperatures up to 164 K. Now, however, an iron-based family of superconductors (pnictides), discovered in 2008, is also vying for attention. In a Perspective on page 272 of this issue ${ }^{3}$, Dimitri Basov and Andrey Chubukov offer their view on the shared properties of the cuprates and pnictides and how such commonalities may help to define the essential ingredients of any high-temperature superconductor.

Reflecting the ongoing activity in the field of superconductivity, this issue also includes several research papers that showcase how the drive to solve the mystery of hightemperature superconductivity has pushed technology to previously unimaginable limits, in the spirit of Kamerlingh Onnes's low-temperature innovations. These include using terahertz spectroscopy to investigate the superconducting fluctuations in $\mathrm{La}_{2-x} \mathrm{Sr}_{x} \mathrm{CuO}_{4}$ (ref. 4); scanning tunnelling microscopy to image bound states within ordered vortices in $\mathrm{Ba}_{0.6} \mathrm{~K}_{0.4} \mathrm{Fe}_{2} \mathrm{As}_{2}$ (ref. 5); and the specific heat of $\mathrm{YBa}_{2} \mathrm{Cu}_{3} \mathrm{O}_{6.56}$ in magnetic fields up to $45 \mathrm{~T}$, which shows quantum oscillations ${ }^{6,7}$. On the theory side, there's also a paper ${ }^{8}$ looking at the relative contribution of spin and orbital polarization to the magnetic state in $\mathrm{BaFe}_{2} \mathrm{As}_{2}$.

But continuing work on fundamental superconductivity is not the only legacy of the original discovery. Nor is the research concentrated on the high-temperature superconductors. On the contrary, organic superconductors, heavy fermions and ruthenates all continue to hold secrets. Over the past century, many ideas spawned by superconductivity have influenced or directly led to whole new fields of research. These include the study of helium-3, both for its cryogenic applications and multiple superfluid phases - Landau's Fermi liquid theory was originally proposed to explain

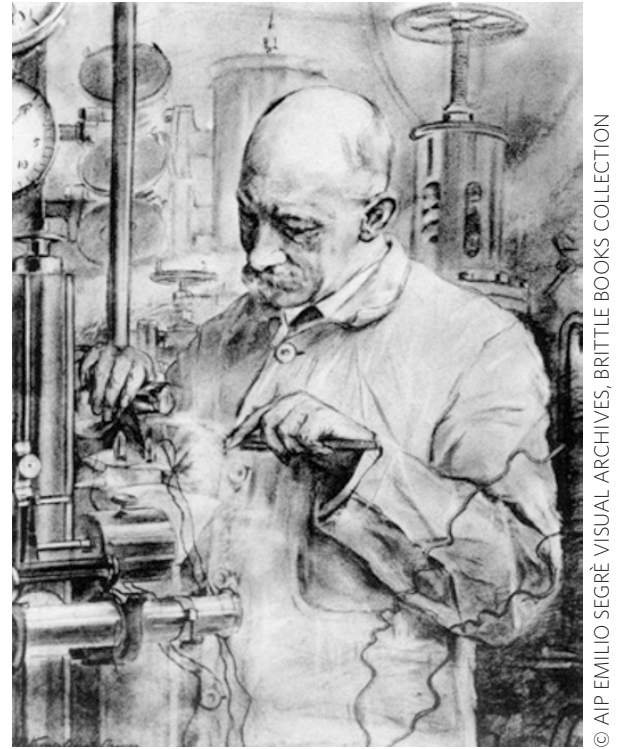

the properties of helium-3. The study of non-Fermi liquids, with several examples of quantum criticality, is another active field.

In fact, these are all examples of strongly correlated electron systems, in which the whole is greater than the sum of its parts. Or, to quote Philip Anderson: "more is different". Such materials exhibit all kinds of unexpected behaviour such as geometric frustration, glassy dynamics and metalinsulator transitions, to name but a few. Work in low-dimensional systems, including the study and manipulation of heterointerfaces within a superlattice structure, is also ongoing; and superconductivity even has an important role to play in the search for Majorana fermions in topological insulators.

Kamerlingh Onnes' determination paid off. A century later, we should face the challenge of building a thorough understanding superconductivity just as determinedly.

\footnotetext{
References

1. Kamerlingh Onnes, H. Nobel Prize lecture (1913); http:// go.nature.com/3AioS9

2. www.nature.com/nmat/focus/superconductivity

3. Basov, D. N. \& Chubukov, A. V. Nature Phys 7, 272-276 (2011).

4. Bilbro, L. S. et al. Nature Phys. 7, 298-302 (2011).

5. Shan, L. et al. Nature Phys. 7, 325-331 (2011).

6. Riggs, S. C. et al. Nature Phys. 7, 332-335 (2011).

7. Tesanovic, Z. Nature Phys. 7, 283-284 (2011).

8. Yin, Z. P., Haule, K. \& Kotliar, G. Nature Phys. 7, 294-297 (2011)
} 